\title{
Measles surveillance in Qatar, 2008: quality of surveillance data and timeliness of notification
}

\author{
Z.A. Nazzal, ${ }^{1}$ H. Said" and N.A. Horeesh ${ }^{7}$
}

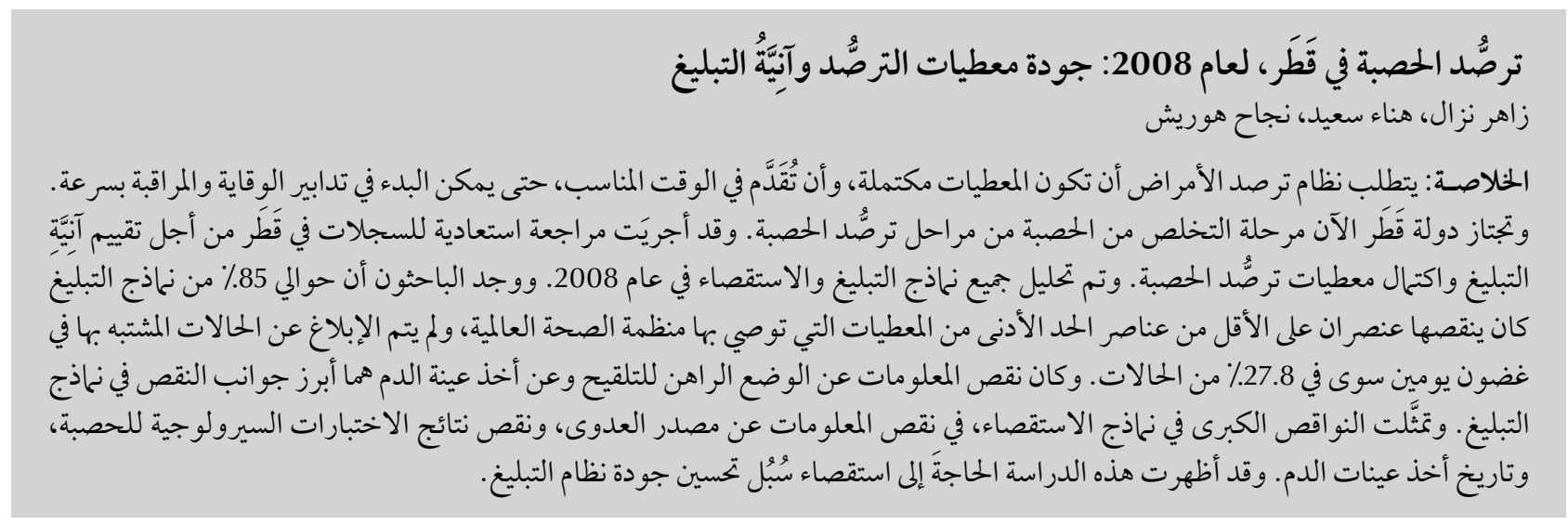

ABSTRACT Disease surveillance systems require that data are complete and submitted on time so that effective prevention and control measures can be initiated promptly. Qatar is in the elimination phase of measles control. A retrospective review of records was conducted in Qatar to evaluate the timeliness of notification and completeness of measles surveillance data. All the notification and investigation forms for the year 2008 were analysed. About $85 \%$ of the notification forms were missing at least 2 of the WHO recommended minimum data elements, and only $27.8 \%$ of the suspected measles cases were reported within 2 days. Lack of vaccination status information and blood specimen collection were the most prominent defects in the notification forms. The major deficiency in the investigation forms were information on the source of infection, measles serology results and date of blood specimen collection. Ways to improve the quality of the notification system need to be investigated.

Surveillance de la rougeole au Qatar en 2008 : qualité des données de surveillance et ponctualité des notifications

RÉSUMÉ Les systèmes de surveillance des maladies nécessitent que les données soient exhaustives et soumises de manière ponctuelle afin d'appliquer rapidement des mesures de lutte efficaces. Le Qatar se trouve à la phase d'élimination dans la lutte contre la rougeole. Un examen rétrospectif des dossiers a été réalisé dans le pays pour évaluer la ponctualité des notifications et l'exhaustivité des données de surveillance de la rougeole. Tous les formulaires de notification et d'analyse pour l'année 2008 ont été analysés. Au moins deux informations de base selon les recommandations de l'Organisation mondiale de la Santé manquaient sur environ $85 \%$ des formulaires de notification et seuls $27,8 \%$ des cas suspects de rougeole avaient été notifiés dans les deux jours. Les informations manquantes sur le statut vaccinal et l'absence de prélèvements d'échantillon de sang étaient les manquements les plus importants dans les formulaires de notification. Les principales lacunes des formulaires d'analyse concernaient la source de l'infection, les résultats sérologiques pour la rougeole et la date du prélèvement de l'échantillon de sang. Des méthodes permettant d'améliorer la qualité du système de notification sont à rechercher. 


\section{Introduction}

Measles is an important infectious disease in humans and has caused millions of deaths since its emergence [1]. It is targeted for elimination in the Eastern Mediterranean Region by 2010 [2]. According to the World Health Organization (WHO) classification, Qatar is in the elimination phase of measles surveillance which requires the country to notify and investigate each suspected measles case immediately.

Surveillance is arguably the most important public health tool used to prevent and control communicable diseases. Surveillance data are essential to establish the need for public health action and assess the effectiveness of prevention programmes $[3,4]$. They should be valid and complete and submitted on time so that effective prevention and control measures can be initiated promptly $[2,5-7]$. The completeness of case notification at the level of individual data elements affects the usefulness of routinely collected data [8]. Completeness of measles surveillance data requires that all the elements that have been defined as the minimum required are reported for each suspected case of measles [9].

These keyattributes ofthe quality of a surveillance system — completeness and timeliness of reporting — should be evaluated periodically $[3,5,10,11]$. A review of the literature revealed previous studies which showed deficiencies in the completeness and timeliness of communicable disease notifications by general practitioners in other countries $[12,13]$. The main aim of the present study in Qatar was to evaluate the measles notification and investigation forms for timeliness of notification and completeness of data in order to improve the quality of the surveillance system towards the ultimate goal of elimination of measles.

\section{Methods}

\section{Setting and sample}

A retrospective records review was conducted in the surveillance unit at the Supreme Council of Health of Qatar to analyse all the measles investigation and notification forms for the year 2008 for timeliness of notification and completeness of data. Ethical approval for the study was obtained from the research committee of HMC.

Data collection and analysis was conducted by the principle investigator between January and February 2009. A total of 234 notification forms and 107 investigation forms for suspected cases of measles for the year 2008 were analysed for availability and legibility of data. These constituted $45.7 \%$ of all notified measles cases in the year 2008 .

\section{Data collection}

A data abstraction sheet for each of the 2 types of forms was constructed based on the $\mathrm{WHO}$ recommendations on the minimum data elements to be filled in the notification and investigation forms [5]. The data elements analysed from the notification forms were: date of birth, geographical area, rash onset, vaccination history and date of last measles vaccination, and date of blood specimen collection. The data elements analysed from the investigation forms were: case information, date of birth, vaccination history and date of last measles vaccination, date of case investigation, geographical area, source of infection, date of onset of rash, date of blood specimen collection, results of measles serology, and date of notification.

The data elements were evaluated based on their availability and legibility. An element was considered available if it was written in the form and considered legible if it was clear and readable. All the forms were evaluated by single person (the principle author) and a variable was judged legible if it was clear and readable by the researcher. To confirm reproducibility of the grading, $10 \%$ of the forms were double-checked by the same person. Timeliness was evaluated by calculating the time in days between the date of rash onset and the date of registration by the central surveillance unit [5]. Elements were scored as available and legible (score 1) or unavailable and/or illegible (score 0 ).

The age of notified cases was divided into 3 groups: $\leq 5$ years, $6-15$ years and $>15$ years The reporting sites were classified into 4 groups: primary health care centres (PHCs); Hamad Medical Corporation (HMC) paediatric department; other HMC departments; and Qatar Petroleum (QP) health care centres/other private health centres. PHC is a government provider of primary care (and some secondary care) through 22 health care centres distributed across the country. HMC is the main government provider of hospital care for all residents in Qatar free of charge. QP is the largest oil company in Qatar, providing primary and secondary health care services to its workers and their families.

\section{Data analysis}

The data abstraction sheets were coded and data entry and analysis was carried out using SPSS, version 16. The proportions of available and legible data elements in the notification and investigation forms and the timeliness of notification were calculated. The chi-squared test was used to compare the availability and legibility of the information on vaccination history and blood specimens with some of the available variables (by reporting facility and age of the reported cases). A $P$-value of 0.05 was used to determine statistical significance.

\section{Results}

\section{Notification forms}

The most prominent deficiency found in the notification forms was a lack of 
information on vaccination history and blood specimen collection. These were available and legible in only $9.4 \%$ and $20.9 \%$ of the notification forms respectively. The most reported data elements were date of birth and rash onset, which were available and legible in $98.3 \%$ and $97.0 \%$ of the forms respectively (Table 1). Overall $85.0 \%$ of notification forms missed at least 2 of the 6 minimum data elements.

The median time between case identification and notification was 4 days, ranging from 1 to 67 days. The percentage of suspected cases reported within 2 days, as recommended by WHO, was $27.8 \%$, while $43.6 \%$ were reported between $>2$ and $\leq 7$ days and $28.6 \%$ more than 7 days after disease onset.

When analysing the results by facility, it was found that $33.3 \%$ of the notification forms from PHC centres were found to have legible information on vaccination history compared with $6.9 \%-17.9 \%$ for forms from other facilities $\left(\chi^{2}=12.69\right.$, $\left.\mathrm{df}=3, P=0.005\right)$ (Table $2)$. A significantly greater proportion of the forms reported from QP health care centres $(50.0 \%)$ contained legible information on blood specimen collection compared with other facilities $(13.3 \%-17.5 \%)\left(\chi^{2}=15.95, \mathrm{df}=3, P=\right.$ $0.001)$.

\begin{tabular}{lcc}
\hline $\begin{array}{l}\text { Table } 1 \text { Availability and legibility of minimum data elements recorded in the } \\
\text { measles notification forms }(\boldsymbol{n}=\mathbf{2 3 4})\end{array}$ & Element available and legible \\
\hline Data element & No. & 9.4 \\
Vaccination history & 22 & 20.9 \\
Blood specimen & 49 & 79.5 \\
Contact information & 186 & 87.2 \\
Geographical area & 204 & 97.0 \\
Rash onset & 227 & 98.3 \\
Date of birth & 230 & \\
\hline
\end{tabular}

Reports of cases among patients aged $>15$ years of age were significantly more likely to have legible information on the date of blood specimen collection than the reports for other age groups $(32.7 \%$ versus $22.0 \%$ and $12.5 \%)\left(\chi^{2}=\right.$ 7.13, $\mathrm{df}=2, P=0.028)$ (Table 2).

\section{Investigation forms}

The most prominent deficiency in the investigation forms was information on the source of infection, measles serology results and date of blood specimen collection. These elements were available and legible in only $13.1 \%, 34.6 \%$ and $46.7 \%$ of the investigation forms respectively (Table 3)

\section{Performance indicators}

Of the WHO recommended performance indicators, 5 of them could be estimated from this study. None of them reached the $\mathrm{WHO}$ recommended standard of $\geq 80 \%$; they ranged from $9.0 \%$ for notification forms with complete information to $45.7 \%$ for the percentage of suspected cases which were investigated (Table 4).

\section{Discussion}

Surveillance data should be valid and complete and submitted on time in order for appropriate prevention and control measures to be initiated. Availability of information on age and vaccination status allows in-depth analysis of surveillance data.

In this study in Qatar a lack of vaccination status information was found to be the most prominent defect in the

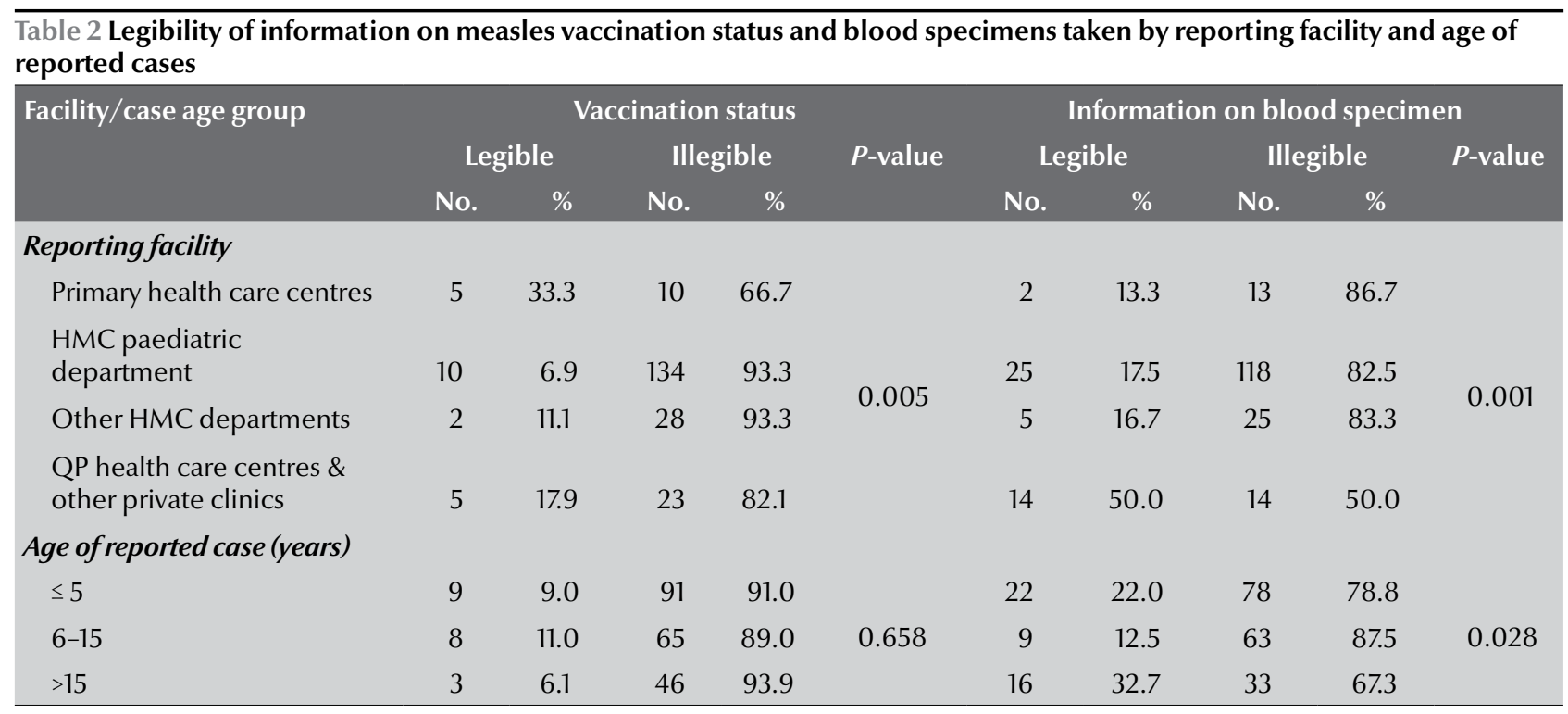

HMC = Hamad Medical Corporation $; P=$ Qatar Petroleum . 


\begin{tabular}{lcc}
\hline $\begin{array}{l}\text { Table } 3 \text { Availability and legibility of minimum data elements recorded in the } \\
\text { measles investigation forms }(\boldsymbol{n = 1 0 7})\end{array}$ & Element available and legible \\
\hline Data element & No. & 13.1 \\
\hline Source of infection & 14 & 34.6 \\
Serology results & 37 & 46.7 \\
Blood specimen collection & 50 & 65.4 \\
Rash onset & 70 & 80.4 \\
Vaccination history & 86 & 91.6 \\
Patient contacts & 98 & 93.5 \\
Geographical area & 106 & 99.0 \\
Date of birth & 106 & 99.0 \\
Contact information & 106 & 99.0 \\
Date of case investigation & 106 & 100.0 \\
Date of notification & 107 &
\end{tabular}

notification forms. Information on vaccination status is very important as it enables public health staff to detect, and then to plan to rectify, possible flaws in the immunization programmes, whether related to coverage or effectiveness of vaccination. Unfortunately, this opportunity was largely missed since the information was recorded in less than $10 \%$ of the notification forms. This figure was lower than the results from Mozambique where vaccination status was recorded in $36 \%$ of cases [14].

Poor recording can be attributed to patients omitting to bring their vaccination card to the consultation, language barriers - a majority of our reported cases (60\%) were among non-Arabic/ non-English speakers - and/or health care workers' lack of awareness about the importance of such information.

Regarding the date of birth of the notified cases, this study showed that age was recorded in $97 \%$ of all reviewed notification forms compared with $73 \%$ reported by Jani et al. in their study in Mozambique [14].

The other major deficiency in completion of the notification forms was related to information on blood specimen collection. Although it is recommended for countries in the measles elimination phase to draw a blood sample as soon as the patient is seen by his/her doctor
[8], only $20.9 \%$ of the reports had information on blood samples, which is considerably lower than the officially adopted performance indicator $(\geq 80 \%$ of the suspected cases should have serological testing).

Case information (patient name, address and telephone number) is very important in notification, as it helps the staff in the central surveillance unit to reach the patient and instigate preventive measures for the contacts and the community. Despite relatively high reporting of this information (on $75 \%$ of the notification forms), much of this information was found to be invalid; either no answer was received at the given contact number or the patient was unavailable at the given address, which made it difficult for the central level staff to contact these patients.

Timely notification of measles is essential for case investigation, as contact tracing is an essential component of an effective surveillance programme. This study shows that only $27.8 \%$ of cases were reported to the central surveillance unit within 2 days of the onset of rash. The median time for reporting a suspected measles case was 4 days. This is in agreement with reports from the United States [15], which showed the median time of measles notification to be 4 days (range 1-66 days), and is much better than achieved in Switzerland [16] where a median of 13 days was reported. However, it is still far from the $\mathrm{WHO}$ recommendations that at least $80 \%$ of suspected cases should be reported within 48 hours. This delay in notification can be due to routine administrative procedures, uncertainty of the proper notification procedures and/or lack of communication between the ministry of health and the health facilities [author's personal observation].

The study results showed that only $45.7 \%$ of the reported cases were investigated. Additionally, most of these were investigated by telephone without home visits. This falls short of the adopted performance indicators ( $\geq 80 \%$ of reported cases should be investigated, mainly through home visits). The lack of home visits and use of phone calls for conducting case and contacts investigation is mainly due to a shortage of personnel at the Qatar central surveillance unit. This is compounded by the inability to contact patients or their families due to invalid telephone numbers or language barriers.

The most common missing data elements in the investigation form was information on blood specimen collection and measles serology results. Although most notified cases did not have blood specimens drawn by the physicians at the peripheral level, a majority of investigation forms completed by the surveillance officer also missed such information. Blood test results were available on $44.0 \%$ of the forms, which indicates that the central surveillance unit did not compensate adequately for the deficiencies at the peripheral levels. This is mainly due to reliance on subjects' complying with the Communicable Diseases Prevention and Control department request to come for blood testing when called for investigation, which is in part of the reason for the WHO recommendation to conduct home visits rather than telephone investigations. 


\section{Conclusion}

In conclusion, the study showed that the notification practices for measles in Qatar were incomplete and were not performed within the recommended timescale. The usefulness of the reporting system was likely to be diminished because of incomplete, absent or incorrect personal data about patients, and the absence of some disease data such as previous vaccination and date of collecting blood samples. Ways to improve the quality of the notification system need to be investigated.

\section{References}

1. Moss WJ, Griffin DE. Global measles elimination. Nature Reviews Microbiology, 2006, 4:900-908.

2. Guris D. Module on best practices for measles surveillance. Geneva, World Health Organization, 2001.

3. Communicable disease surveillance and response systems: guide to monitoring and evaluating. Geneva, World Health Organization, 2006 (WHO/CDS/EPR/LYO/2006).

4. Centers for Disease Control and Prevention. Updated guidelines for evaluating public health surveillance systems: recommendations from the Guidelines Working Group. Morbidity and Mortality Weekly Report, 2001, 50 No. RR-13;1-35.

5. WHO recommended standards for surveillance of selected vaccine-preventable diseases. Geneva, World Health Organization, 2003 (WHO/V\&B/03.01).

6. Global framework for immunization monitoring and surveillance. Geneva, World Health Organization, 2007.

7. Jajosky R, Groseclose S. Evaluation of reporting timeliness of public health surveillance systems for infectious diseases. BMC Public Health, 2004, 4:29.

8. Pan American Health Organization. Case definitions: measles and rubella. Epidemiological Bulletin, 1999, 20:10-12.

9. Vogt RL et al. Evaluation of the timeliness and completeness of a web-based notifiable disease reporting system by a local health department. Journal of Public Health Management and Practice, 2006, 12:540-544.
10. Wharton M, Ching P. Surveillance indicators. In: Manual for the surveillance of vaccine preventable diseases, 3rd ed. Atlanta, Georgia, Centers for Disease Control and Prevention, 2002:1-15.

11. Hodge $M$, Haghgou $M$, Birmingham M. Making surveillance work. Module 3: logistics management. Geneva, World Health Organization, 2001:22.

12. Gauci $\mathrm{C}$ et al. General practitioners' role in the notification of communicable diseases: study in Malta. Eurosurveillance, 2007, 12(11):E5-E6.

13. Seneviratne S, Gunatilake S, Silva H. Reporting notifiable diseases: methods for improvement, attitudes and community outcome. Transactions of the Royal Society of Tropical Medicine and Hygiene, 1997, 91:135-137.

14. Jani J et al. Assessment of routine surveillance data as a tool to investigate measles outbreaks in Mozambique. BMC Infectious Diseases, 2006, 6:29.

15. Harpaz R et al. Lessons learned from establishing and evaluating indicators of the quality of measles surveillance in the United States, 1996-1998. Journal of Infectious Diseases, 2004, 189(s1):S196-S203.

16. Richard JL, Vidondo B, Mäusezahl M. A 5-year comparison of performance of sentinel and mandatory notification surveillance systems for measles in Switzerland. European Journal of Epidemiology, 2008:23(1):55-65. 